\title{
Influence of leading edge imperfections on the aerodynamic characteristics of NACA 63,-2 15 laminar aerofoils at low Reynolds numbers
}

\author{
L Ayuso', R Sant' and J Meseguer ${ }^{2}$
}

\begin{abstract}
This article deals with the effect of leading edge imperfections on the aerodynamic characteristics of a NACA $63_{2}-215$ laminar aerofoil at low Reynolds numbers. Wind tunnel tests have been performed at different Reynolds numbers and angles of attack and global aerodynamic loads were measured. To perform these tests, a NACA 63 $2-215$ aerofoil was built up in two halves (corresponding to the upper side and to the lower side), the leading edge imperfection here considered being a slight displacement of half aerofoil with respect to the other. From experimental results, a quantitative measure of the influence of the leading edge displacement on the degradation of the aerofoil aerodynamic performances has been obtained. This allows the establishment of a criterion for an acceptance limit for this kind of imperfection.
\end{abstract}

\section{Keywords}

Leading edge, low Reynolds number, laminar aerofoil

\section{Introduction}

In this article the influence of leading edge imperfections on the aerodynamic performances of a classical laminar aerofoil like NACA $63_{2}-215$ is considered. Leading edge imperfections can arise during the manufacturing process, mainly in non-aeronautical applications, where manufacturing methods are not so strict, than in aeronautical applications. A typical example can be found in wind turbine blades, which are usually manufactured in two parts, or shells (the upper surface and the lower surface), and after assembled. However, in some cases, this last step is not performed properly and some displacement between the edges of the shells results, which could affect the aerodynamic behaviour of the aerofoil.

Assuming the leading edge displacement is small enough, this kind of imperfection can be corrected by reducing the leading edge imperfection through refilling and sandpaper treatment, which requires considerable manpower which in turn leads to increase of production costs. Obviously, a modification of leading edge geometry like the one considered here means some deterioration of the aerodynamic performances of the resulting aerofoil. However, despite the relative importance of this problem, the influence of this leading edge imperfection on the aerodynamic performance is not very well documented in the literature.
Low Reynolds number aerofoils have been the focus of attention of many researchers over the decades because of their use in a wide range of applications, from unmanned aerial vehicles to low-power wind turbine blades and also because of their potential use in aircrafts designed to fly in thin atmospheres like the one existing in Mars. ${ }^{18}$

In the case of symmetric non-laminar aerofoils at low Reynolds numbers, leading edge imperfections like the one considered here introduce a loss of symmetry in the aerofoil shape, which causes a lift coefficient increase in some cases (in symmetric aerofoil the leading edge displacement is similar to a camber effect) and an important rise of the drag coefficient, ${ }^{9,10}$ thus leading to a significant reduction of the aerodynamic efficiency. Similar results are obtained with leading edge flaps at low Reynolds numbers. ${ }^{11}$

\footnotetext{
'Departamento de Aerotecnia, EUIT Aeronáutica, Universidad Politécnica de Madrid, Madrid, Spain

'Instituto Universitario 'Ignacio Da Riva' (IDR/UPM), Universidad Politécnica de Madrid, Madrid, Spain
}

\section{Corresponding author:}

L Ayuso, Departamento de Aerotecnia, EUIT Aeronáutica, Plaza del Cardenal Cisneros, 3, Universidad Politécnica de Madrid, 28040 Madrid, Spain.

Email: luis.ayuso@upm.es 


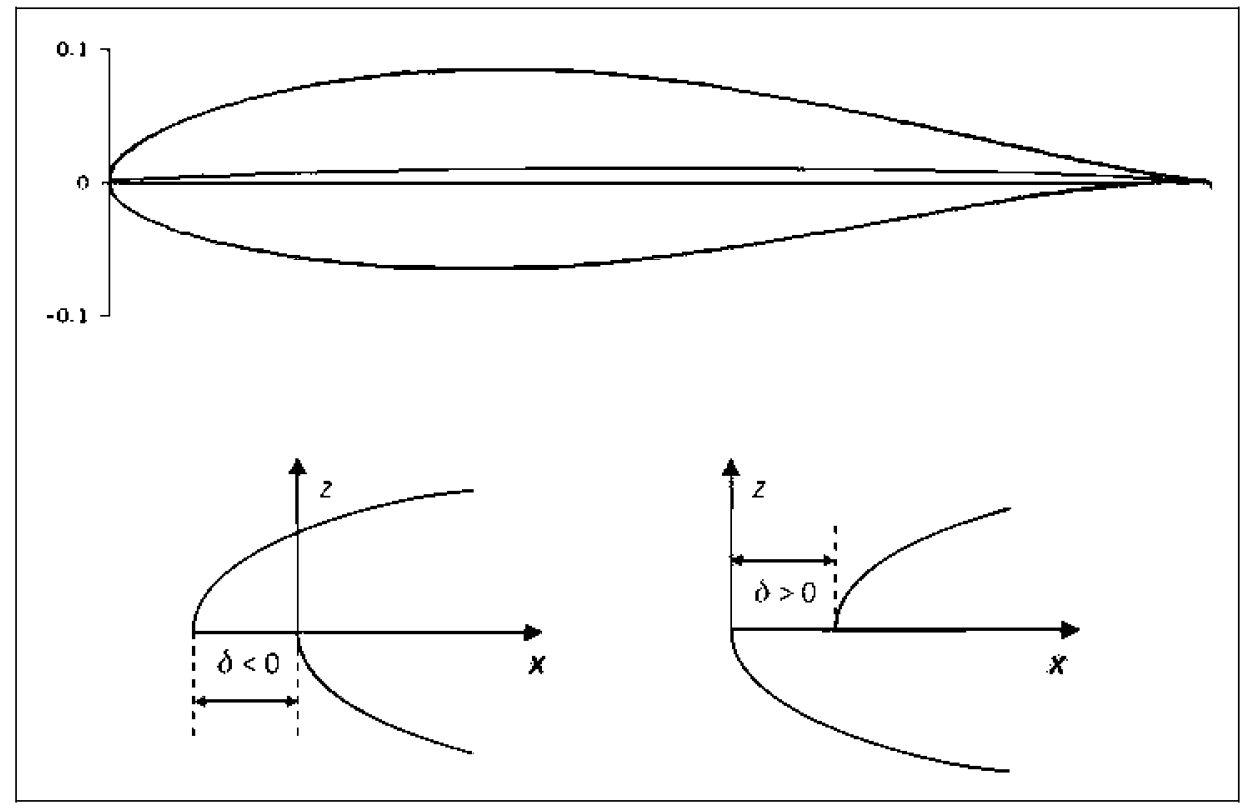

Figure I. Sketches of the nominal NACA $63_{2}-215$ model and of the modified ones by leading edge perturbations: the upper surface is displaced a distance $x_{d}$ with respect to the lower surface. The distance $x_{d}$ is assumed to be positive when the edge of the aerofoil upper side is behind the edge of the lower one $(A)$ and negative when the edge of the upper side is in front of the edge of the lower side $(\mathrm{B})$.

When cambered aerofoils are considered, the behaviour is not so clear because the camber effect associated with leading edge displacement can act in the same sense or in opposition to the geometrical aerofoil camber, amongst other effects.

Aiming to gain additional insight regarding this phenomenon, a test campaign was carried out to measure the influence of a displacement between the leading edges of the upper and lower surfaces on aerodynamic performances. Measurements were performed at low Reynolds numbers by using a laminar aerofoil NACA $63_{2}-215$ (Figure 1). The aerofoil model was built up in two half parts (the upper and the lower one), which can be displaced one with respect to the other; therefore, the basic aerofoil shape can be modified by modifying the dimensionless distance $\delta=x_{d} / c$ between the leading edges of the upper and lower part of the aerofoil (here, $x_{d}$ is the distance between both edges and $c$ is the nominal aerofoil chord). Experimental results show that the degradation of aerofoil performances becomes unacceptable outside the range $-0.005 \leqslant \delta \leqslant 0.010$.

\section{Experimental setup}

Experiments were carried out at the Departamento de Aerotecnia of the Universidad Politécnica de Madrid. A small open-circuit low-speed wind tunnel was used. The wind tunnel test chamber is $1.2 \mathrm{~m}$ high, $0.16 \mathrm{~m}$ wide and $1.5 \mathrm{~m}$ long. The test chamber is equipped with two windows (at the lateral walls of the test chamber), one of them being optically transparent. Upstream of the test section is the contraction, with several screens at the contraction entrance, which provide a uniform low-turbulent incoming flow at the test chamber entrance.

Flow uniformity in the test chamber, outside boundary layers, is less than $1 \%$ and the mean turbulence level is less than $0.5 \%$. Flow uniformity is defined as the ratio of the difference between the maximum, $U_{\max }$, and minimum, $U_{\min }$, velocities and the mean velocity, $U_{\text {mean }}$, that is $\left(U_{\max }-U_{\text {min }}\right) / U_{\text {mean }}$. Air velocity in the test section ranged from 5 to $30 \mathrm{~m} / \mathrm{s}$. Thus, based on nominal aerofoil chord, $c=0.24 \mathrm{~m}$, Reynolds numbers up to $4.5 \times 10^{5}$ were reached.

A model of a laminar aerofoil NACA $63_{2}-215^{12}$ was used. The model has been manufactured in a numerically controlled milling machine using an isotropic material widely used in modelling (Necuron ${ }^{\circledR}$ ), which allows a good aerofoil surface finish. The aerofoil sample is equipped with a simple mechanism that permits the relative displacement of upper side of the aerofoil with respect to the lower one, which is the one anchored to the wind tunnel reference frame.

Model span is $15.8 \mathrm{~cm}$, whereas that of the wind tunnel test chamber is $16 \mathrm{~cm}$. No special provisions were undertaken to avoid air flow through the gap between the model and wind tunnel walls. Since the aim of these experiments was to quantify the relative degradation of the aerofoil due to leading edge imperfections, this gap has not been taken into account to correct measured results (in any case, its effect is assumed to be negligible).

In order to verify that this assumption is correct, during the wind tunnel characterization tests, some prior measurements were performed using a 


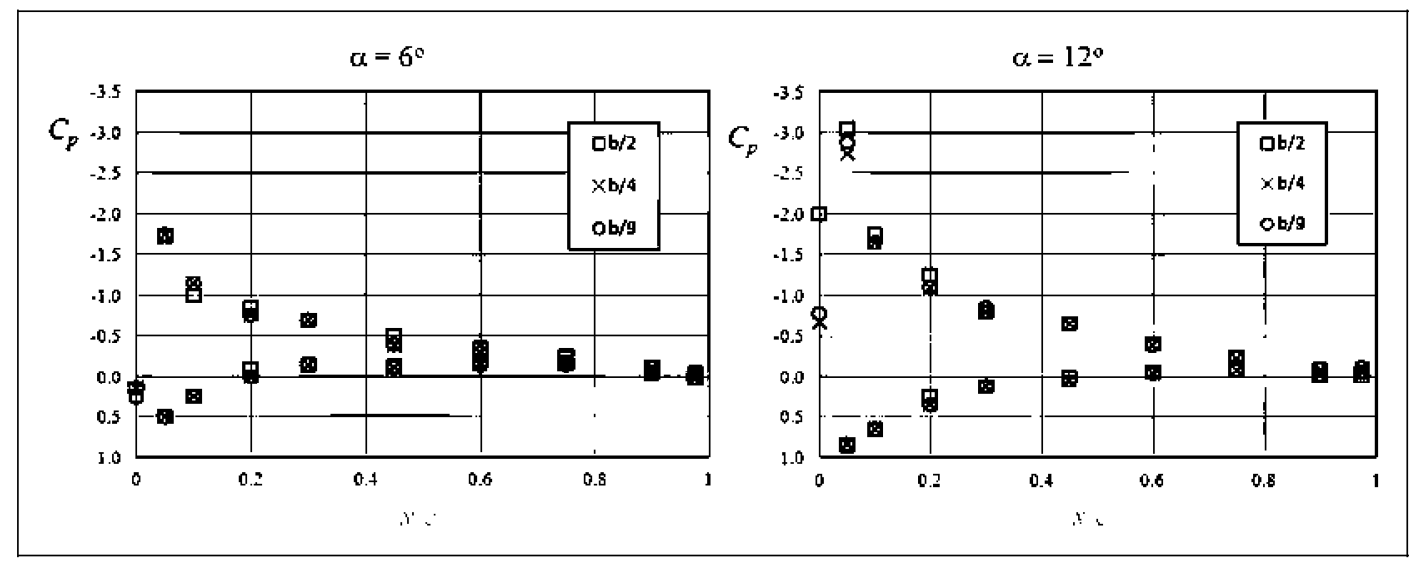

Figure 2. Measured pressure distributions on NACA 0012 aerofoil for $\alpha=6^{\circ}$ and $\alpha=12^{\circ}$. Re $=2 \times 10^{5}$. Symbols identify the value of the wing section according to the following key: $y=b / 2$ (white squares); $y=b / 4$ (black cross) and $y=b / 9$ (white circles).

symmetric aerofoil model equipped with pressure taps. The model is a NACA $0012^{12}$ equipped with 57 pressure taps distributed in three sections, located at $y / b=1 / 9,1 / 4$ and $1 / 2$, where $y$ is the distance from one of the model tips and $b$ is the model span $(b=15.8 \mathrm{~cm})$. At each measuring section, there are nine pressure taps on the upper surface, another nine pressure taps on the lower surface plus one additional pressure tap at the leading edge. Pressure distributions were measured and recorded at $125 \mathrm{~Hz}$ sampling rate for a period of $10 \mathrm{~s}$. For each aerofoil configuration (angle of attack and Reynolds number), the values of the coefficients used to present experimental results are the averaged values of those obtained in the whole measurement period. This model was used to evaluate the two-dimensional character of the flow past the aerofoil, as it was demonstrated from the comparison of measured pressure distributions at different span locations, where no significant differences were found (Figure 2).

Since the aerofoil chord is less than 0.25 times the height of the test chamber and the angle of attack $\alpha$ has been kept within the range $|\alpha|<24^{\circ}$, no blockage corrections were performed. ${ }^{13} 15$ It must be emphasized that the aim of this study is mainly to compare the effect of different aerofoil leading edge imperfections on aerodynamic performances.

During tests, the angle of attack ranged from $-2^{\circ}$ to $24^{\circ}$. Four values of Reynolds number were considered: $0.75 \times 10^{5}, 1.5 \times 10^{5}, 3.0 \times 10^{5}$ and $4.5 \times 10^{5}$.

Aerodynamic loads were measured with a threecomponent electronic balance from PLINT Company. The balance is located at one of the side walls of the test chamber. Measured loads were lift, drag and pitching moment. The electronic balance load range is $100 \mathrm{~N}$ in lift forces, $50 \mathrm{~N}$ in drag forces and $3.1 \mathrm{Nm}$ in torque and its accuracy is $0.015 \mathrm{~N}$ in lift forces, $0.0076 \mathrm{~N}$ in drag forces and $4.8 \times 10^{-4} \mathrm{Nm}$ in torque. At each angle of attack, loads were measured at $10^{3} \mathrm{~Hz}$ sampling rate during $8 \mathrm{~s}$. Repeatability is good enough and uncertainty is low enough, obtaining values RMS of root mean square of 0.004 for lift coefficient, 0.002 for drag coefficient and 0.001 for pitch moment coefficient.

As already said, upper and lower aerofoil surfaces are separated so that any desired leading edge displacement can be reached (Figure 1). Note that when a displacement is established at the leading edge, the resulting aerofoil is not exactly a NACA $63_{2}-215$ but a similar one with slightly different values and locations of the maximum chamber and maximum thickness, depending on the value of the leading edge displacement, although the differences with the nominal aerofoil at the leading edge become remarkable (Figure 3 ).

It has been stated that positive leading edge displacements are reached when the upper side edge is behind the lower-half edge and negative in the contrary case (Figure 1). The tested dimensionless leading edge displacements, $\delta=x_{d} / c$, were $\delta=0, \pm 0.0025$, $\pm 0.0050, \pm 0.0075, \pm 0.0100$ and \pm 0.0150 .

The measured loads have been made dimensionless by using the aerofoil chord, $c \delta=c(1+\delta)$ (where $c$ is the chord corresponding to $\delta=0$ ), and the upstream flow dynamic pressure, $q_{\infty}=\rho U_{\infty}^{2} / 2$, as characteristic magnitudes, where $\rho$ is the air density and $U_{\infty}$ stands for the upstream flow speed. From load measurements, the variation with the aerodynamic angle of attack of the lift coefficient, $C_{l}=l /\left(q_{\infty} c \delta\right)$, the drag coefficient, $C_{d}=d /\left(q_{\infty} c \delta\right)$, the pitch moment coefficient, $C_{m}=m /\left(q_{\infty} c_{\infty}^{2}\right)$, as well as the lift to drag ratio, $\eta=C_{l} / C_{d}$, have been obtained. Here $l, d$ and $m$ are the lift, drag and pitching moment per unit span length, respectively.

Note that, as already stated, when $\delta \neq 0$, the aerofoil chord is not exactly equal to the nominal aerofoil chord, $c$, which affects the definition of the angle of attack. Because of that, the aerodynamic angle of attack was used instead of the geometrical angle of attack. Aerodynamic angle of attack is defined as the angle between upstream flow direction and aerofoil zero lift direction. 


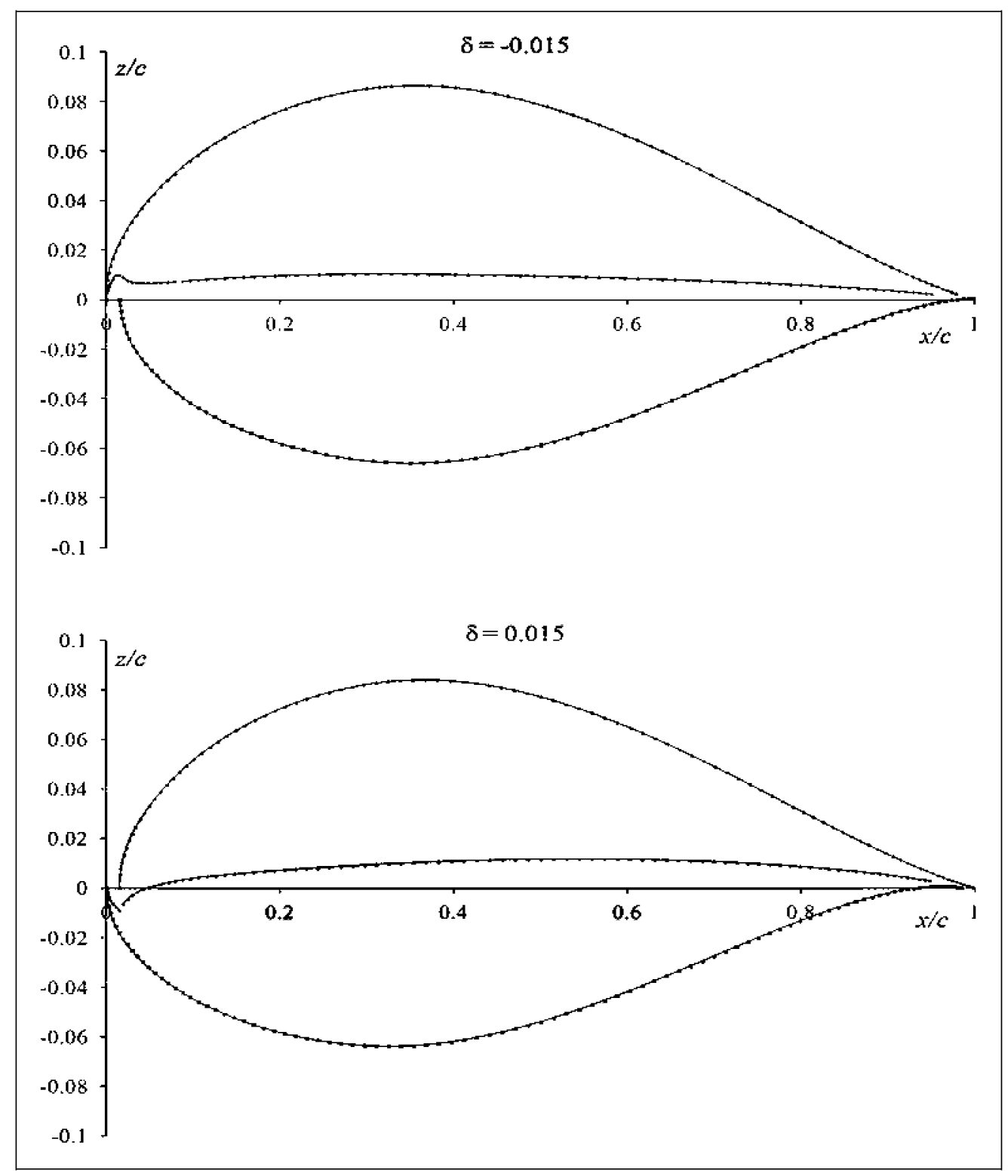

Figure 3. Sketches of the NACA $63_{2}-215$ aerofoil, showing the camber lines in the cases $\delta=0.015$ and $\delta=-0.015$. Vertical scales are oversized.

\section{Results and discussion}

\section{Nominal aerofoil NACA $63_{2}-215$}

The results regarding the aerodynamic performances of the nominal aerofoil NACA $63_{2}-215$, the variation with the aerodynamic angle of attack of the lift coefficient, the drag coefficient, the pitch moment coefficient at $1 / 4$ chord point and the lift to drag ratio are shown in Figure 4. In these plots, the results corresponding to the above stated Reynolds number values $\left(0.75 \times 10^{5}, 1.5 \times 10^{5}, 3.0 \times 10^{5}\right.$ and $\left.4.5 \times 10^{5}\right)$ have been represented as well as the results corresponding to $\operatorname{Re}=3.0 \times 10^{6}$ (obtained from Abbott and Von Doenhoff ${ }^{12}$ ). Although measurements have been performed both by increasing and decreasing the angle of attack, no hysteresis has been detected, as one can expect, the reason being that the time interval between two consecutive measurements is large enough.
According to experimental results, within the range of values of the Reynolds number of the experiments performed here, as the Reynolds number grows, the nominal aerofoil NACA $63_{2}-215$ slightly increases the lift coefficient, as well as the maximum lift coefficient. The lift curve slope also increases as the Reynolds number increases. In all cases, the linear behaviour of the lift curve changes when the aerodynamic angle of attack becomes large enough: the lift curve slope becomes smaller when the aerodynamic angle of attack is larger than $12^{\circ}$ approximately. This change in the lift slope curve can be explained because of the formation of a recirculation bubble at the upper side aerofoil, close to the leading edge. Close to the above quoted value of the angle of attack, the laminar boundary layer detaches at the leading edge and reattaches to the upper surface aerofoil after the shear layer that bounds the almost potential outer flow 


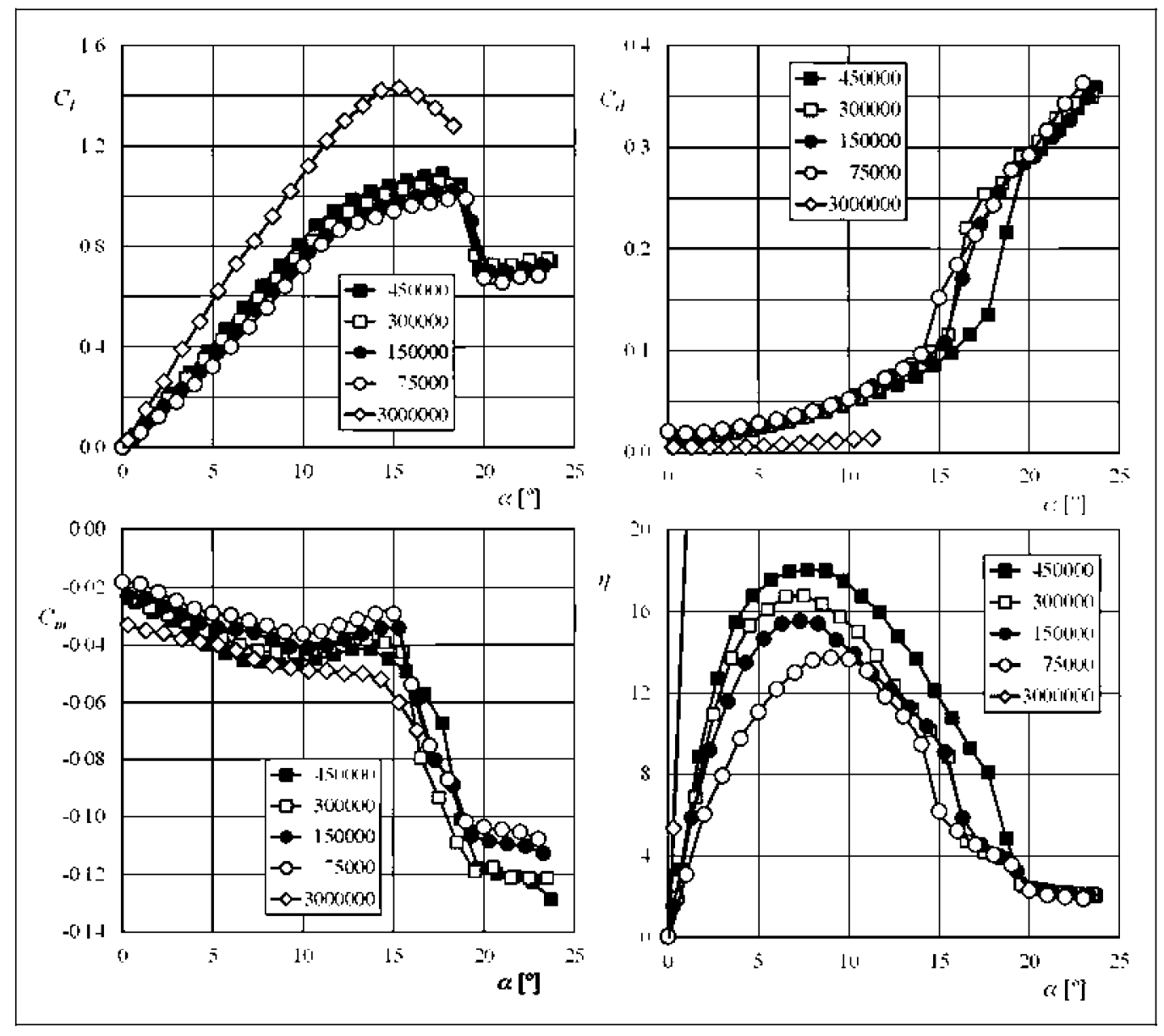

Figure 4. Aerodynamic performances of a NACA $63_{2}-215$ aerofoil. Variation with the aerodynamic angle of attack, $\alpha$, of the lift coefficient, $C_{l}$, drag coefficient, $C_{d}$, pitch moment at $1 / 4$ chord point, $C_{m}$, and efficiency or lift to drag ratio, $\eta=C_{l} / C_{d}$. Symbols identify the value of the Reynolds number according to the following key: $\operatorname{Re}=0.75 \times 10^{5}$ (white circles); $1.5 \times 10^{5}$ (black circles); $3.0 \times 10^{5}$ (white squares); $4.5 \times 10^{5}$ (black squares) and $3.0 \times 10^{6}$ (rhombi).

from the separated one forcing the transition to turbulent flow. The size of the recirculation bubbles increases as the angle of attack increases, until the reattachment point reaches the trailing edge and then, after a threshold value of the angle of attack (around $19^{\circ}$ ), the aerofoil stalls suddenly, independent of the value of the Reynolds number (such a behaviour is similar to the one described in previous stu$\operatorname{dies}^{16,17}$ in the case of thin aerofoils). Additional details on the measured pressure distributions can be obtained upon request to the authors.

The drag coefficient of the nominal NACA $63_{2}-215$ aerofoil decreases as the Reynolds number increases, so that the aerodynamic efficiency (lift to drag ratio) increases, while the angle of attack of maximum efficiency decreases.

On the other hand, the pitching moment at the aerodynamic centre becomes more and more negative as the Reynolds number increases.

It is worthwhile to compare load coefficient measured at low Reynolds number with those resulting at high Reynolds number. In the same plot of Figure 4, the results corresponding to $\operatorname{Re}=3.0 \times 10^{6}$ have been depicted. ${ }^{12}$ Obviously, the aerodynamic performances of this aerofoil are significantly improved, provided the Reynolds number is large enough, except in the case of the stall angle of attack, which becomes larger at low values of the Reynolds number. In any case, the efficiency of NACA $63_{2}-215$ aerofoil at the test Reynolds numbers here considered is very small when compared with the efficiency obtained at $\operatorname{Re}=3 \times 10^{6}$, although such a behaviour is in agreement with the results reported in previous studies: ${ }^{2,4}$ in the range $10^{4} \leqslant \operatorname{Re} \leqslant 10^{6}$, the aerodynamic efficiency of smooth surface aerofoils changes by almost two orders of magnitude.

\section{Effect of leading edge imperfections}

Concerning the influence of both the Reynolds number, Re, and the leading edge displacement, $\delta$, some of the results obtained are shown in Figures 5 and 6 , where the variation of the aerofoil aerodynamic performances (lift coefficient $C_{l}$, drag coefficient $C_{d}$, pitch moment coefficient $C_{m}$ and efficiency $\eta=C_{l} / C_{d}$ ) with the aerodynamic angle of attack, $\alpha$, of aerofoils having different dimensionless leading edge displacement, $\delta$, are presented. For the sake of clarity, 


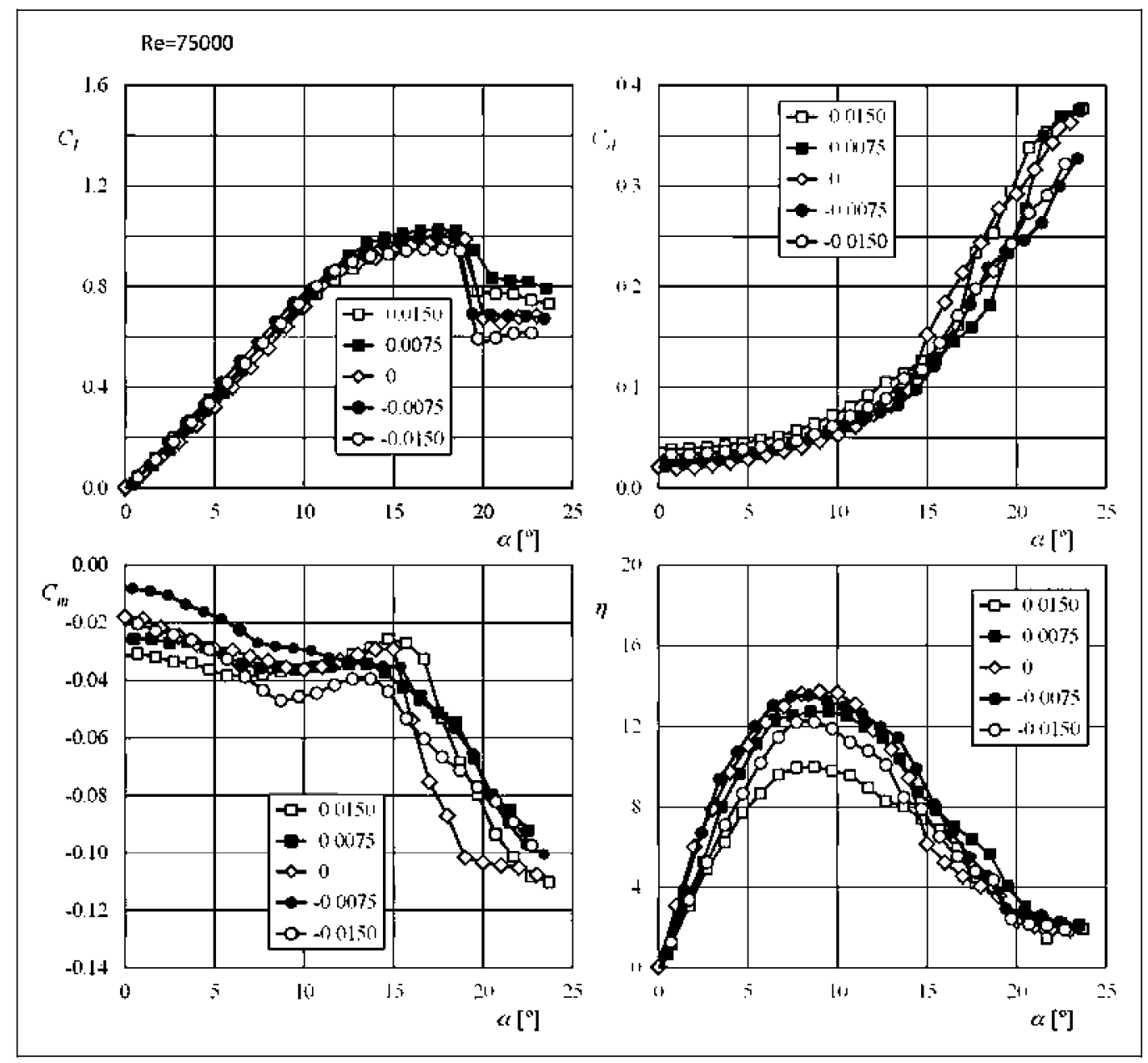

Figure 5. Aerodynamic performances of modified NACA $63_{2}-215$ aerofoils. Variation with the aerodynamic angle of attack, $\alpha$, of the lift coefficient, $C_{b}$, drag coefficient, $C_{d}$, pitch moment at $1 / 4$ chord point, $C_{m}$, and efficiency or lift to drag ratio, $\eta=C_{/} / C_{d}$. Results corresponding to a Reynolds number $\operatorname{Re}=0.75 \times 10^{5}$. Symbols identify the value of the dimensionless leading edge displacement $\delta$ according to the following key: $\delta=0.0150$ (white squares), $\delta=0.0075$ (black squares), $\delta=0$ (white rhombi), $\delta=-0.0075$ (black circles) and $\delta=-0.0150$ (white circles).

only five values of the dimensionless leading edge displacement $\delta$ (instead of the nine measured) are shown in the graphs and only results corresponding to the two extreme values of the tested Reynolds number are presented: $\operatorname{Re}=7.5 \times 10^{4}$ (Figure 5) and $\operatorname{Re}=4.5 \times 10^{5}$ (Figure 6).

As shown in Figures 5 and 6 , when $\delta \neq 0$, the differences with respect to the case $\delta=0$ are not too large, the lift coefficient grows linearly or almost linearly with the angle of attack until $\alpha \cong 12^{\circ}$, where a change of the lift slope curve appears independent of the values of the leading edge displacement, $\delta$. If the angle of attack is increased further, the aerofoils stall and a sudden decrease of the lift coefficient takes place. Experimental results show that the aerodynamic angle of attack to which the upper aerofoil surface becomes completely stalled is almost independent of the leading edge displacement. On the other hand, the aerodynamic drag coefficient increases as the displacement $\delta$ increases, provided the aerodynamic angle of attack is not too large, although the increment of the drag coefficient with the aerodynamic angle of attack is smaller for negative values of $\delta$ (in any case, the impact of the leading edge displacement $\delta$ on the aerodynamic coefficients, lift, drag and pitch moment, is less pronounced as the Reynolds number increases).

Both force coefficients (lift and drag) can be merged into a single one: the aerodynamic efficiency or the lift to drag ratio $\eta=C_{l} / C_{d}$. Concerning the aerodynamic efficiency, independent of the value of the Reynolds number, a displacement, either positive or negative, of the leading edge of the upper surface means some deterioration of the $C_{l} / C_{d}$ ratio, provided the displacement $\delta$ is high enough, although this deterioration is more accentuated for positive values of $\delta$ than for negative ones. According to experimental results, the aerofoil efficiency decreases as the leading edge imperfection grows $(|\delta|>0)$. The maxima of the different $\eta=C_{l} / C_{d}$ curves are reached at similar values of the angle of attack as $\delta$ increases, although the magnitude of these maxima are smaller as $\delta$ grows. With respect to negative displacements $(\delta<0)$, due to the lift increment and the drag decrement obtained 


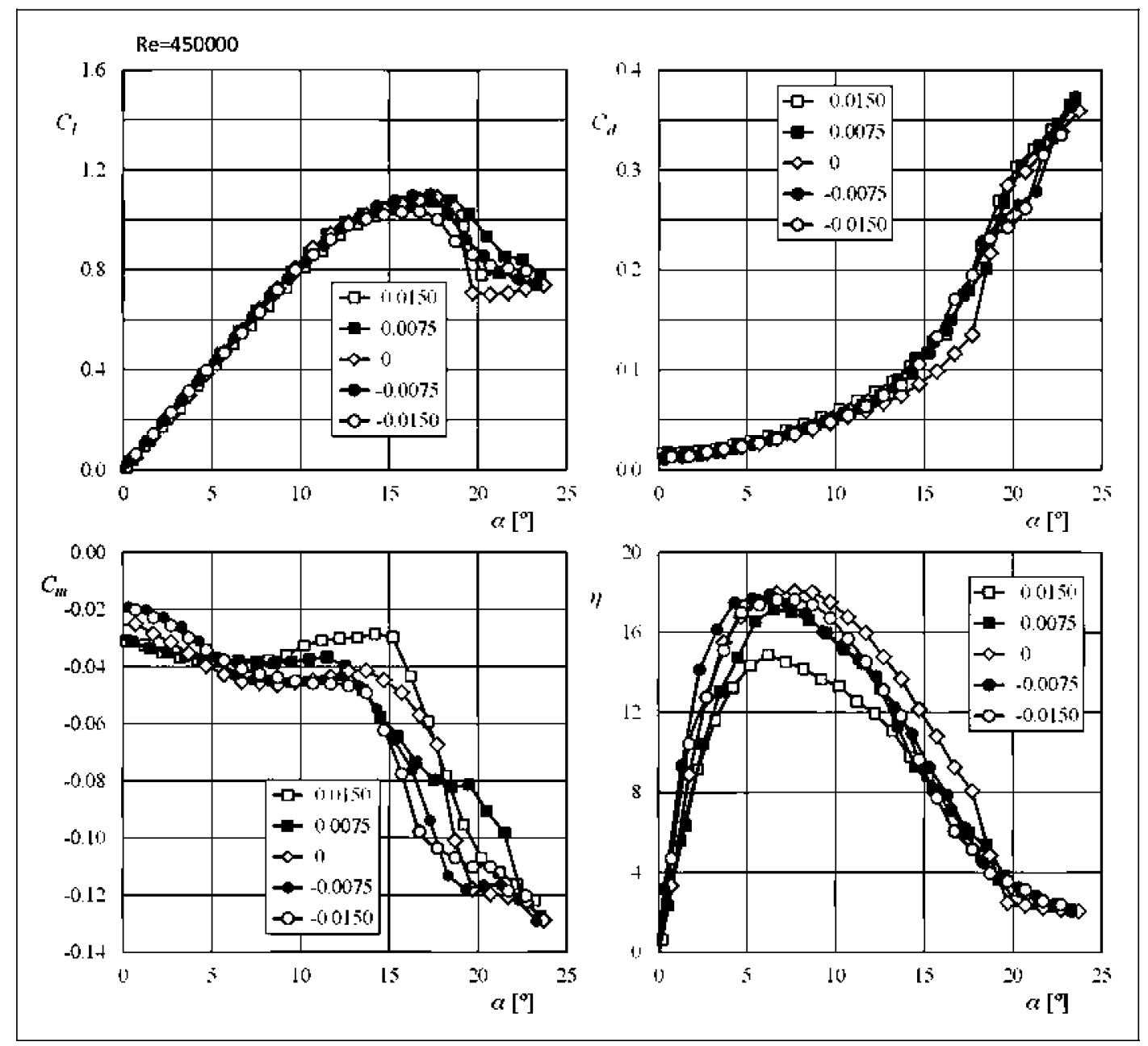

Figure 6. Aerodynamic performances of modified NACA $63_{2}-215$ aerofoils. Variation with the aerodynamic angle of attack, $\alpha$, of the lift coefficient, $C_{l}$, drag coefficient, $C_{d}$, pitch moment at $1 / 4$ chord point, $C_{m}$, and efficiency or lift to drag ratio, $\eta=C_{l} / C_{d}$. Results corresponding to a Reynolds number $\operatorname{Re}=4.5 \times 10^{5}$. Symbols identify the value of the dimensionless leading edge displacement $\delta$ according to the following key: $\delta=0.0150$ (white squares); $\delta=0.0075$ (black squares); $\delta=0$ (white rhombi); $\delta=-0.0075$ (black circles) and $\delta=-0.0150$ (white circles).

for small negative leading edge displacements, aerofoil efficiencies are a little larger when compared with those of positive leading edge displacement. This behaviour is almost the same, independent of the value of the Reynolds number.

Additional insight on the degradation of the aerodynamic performances by leading edge imperfections can be reached from the analysis of some aerodynamic global characteristics like the maximum lift coefficient, $C_{l_{\max }}$, the maximum efficiency, $\eta_{\max }$, or $1 / 4$ chord point pitch moment at zero aerodynamic angle of attack, $C_{m 0}=C_{m}(0)$. With respect to $C_{\text {max }}$, the variation of this parameter with the leading edge deformation $\delta$ is shown in Figure 7. As it can be observed, for each value of the Reynolds number, roughly speaking, the maximum lift coefficient slightly increases as the displacement $\delta$ grows, at least within the range $-0.0075 \leqslant \delta \leqslant 0.0050$, whereas $C_{\text {Imax }}$ values drop outside this interval. Such behaviour can be explained because of the changes of the whole aerofoil geometry due to $\delta$. According to Figure 3, positive values of $\delta$ tend to increase the aerofoil camber, whereas the contrary occurs when $\delta$ is negative, as shown in Figure 8, where the variation with the leading edge displacement $\delta$ of the dimensionless maximum camber ordinate, $\varepsilon_{m} / c$, and the dimensionless position along the chord of this maximum ordinate, $x_{m} / c$, have been depicted. It must be mentioned that the maxima represented in Figure 8 correspond to those located close to the middle point of the aerofoil, since there can be another maxima close to the leading edge, provided the absolute value of the displacement $\delta$ is large enough (see Figure 3).

Thus, according to Figure 8, the maximum of the camber line of modified NACA $63_{2}-215$ aerofoils increases and displaces to the trailing edge as $\delta$ grows. Besides, the displacement $\delta$ causes at the leading edge an anomaly in the camber line, which could be assimilated to a very small leading edge flap, although its effect on the aerodynamic loads does not seem to be important (note that the length of this leading edge flap would be of order $\delta$ ). 


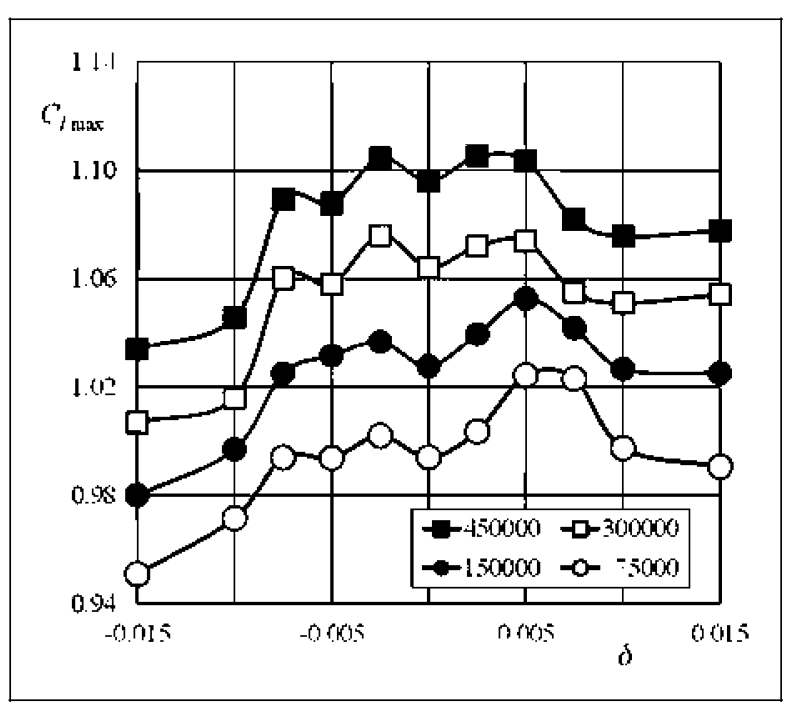

Figure 7. Variation with the leading edge displacement $\delta$ of the maximum the lift coefficient, $C_{\text {Imax }}$, of modified NACA $63_{2}-215$ aerofoils. Symbols identify the value of the Reynolds number according to the following key: $\operatorname{Re}=0.75 \times 10^{5}$ (white circles); I. $5 \times 10^{5}$ (black circles); $3.0 \times 10^{5}$ (white squares) and $4.5 \times 10^{5}$ (black squares).

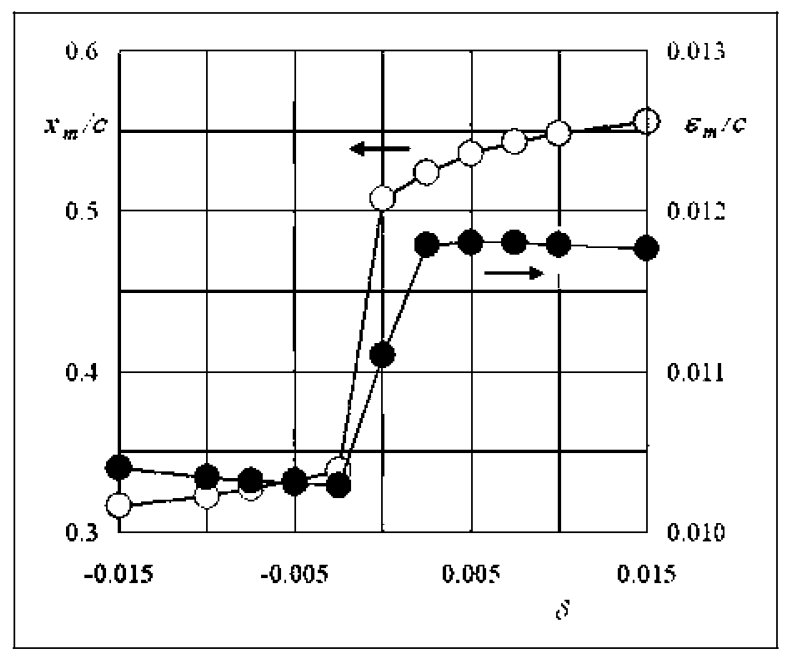

Figure 8. Variation with the leading edge displacement $\delta$ of the dimensionless maximum camber ordinate, $\varepsilon_{m} / c$ (black circles), and the dimensionless position along the chord of this maximum ordinate, $x_{m} / c$ (white circles), of modified NACA $63_{2}-215$ aerofoils.

Concerning the data plotted in Figure 8, as the maximum of the camber line moves towards the trailing edge and its magnitude increases $(\delta>0)$, the lift increases and the aerodynamic centre also moves to the trailing edge. Therefore, the pitch moment becomes more negative than the pitch moment value corresponding to $\delta=0$, and the contrary occurs when $\delta<0$ (the variation with $\delta$ of the pitch moment corresponding to zero values of the aerodynamic angle of attack is presented in Figure 9).

Note that, depending on the value of the Reynolds number, the different $C_{m 0}$ versus $\delta$ curves follow the

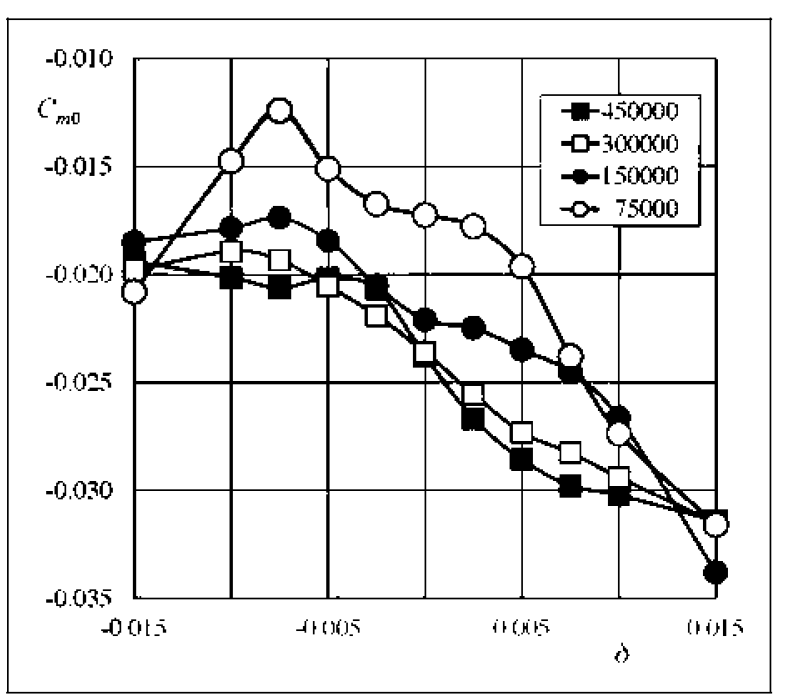

Figure 9. Variation with the leading edge displacement $\delta$ of the pitch moment coefficient corresponding to zero aerodynamic angle of attack, $C_{m 0}$, of modified NACA $63_{2}-215$ aerofoils. Symbols identify the value of the Reynolds number according to the following key: $\operatorname{Re}=0.75 \times 10^{5}$ (white circles); $1.5 \times 10^{5}$ (black circles); $3.0 \times 10^{5}$ (white squares) and $4.5 \times 10^{5}$ (black squares).

above described behaviour until $\delta \cong-0.008$, where the slope of the curves changes its sign (provided Reynolds number is low enough), and then further increments of the upper side leading edge displacement (see Figure 3) causes the pitching moment to be more and more negative. In effect, according to Figure 3, the leading edge displacement $\delta$ can be assimilated to a mean positive camber (almost parabolic) plus a flat plate with a small leading edge flap, deflected upward or downward depending on the values of $\delta$. Assuming a linear behaviour, the deflection $\delta$ does not modify the contribution of the camber to the value of $C_{m 0}$ except close to $\delta=0$, where the camber suddenly decreases as $\delta$ decreases (Figure 8), so that the value of $C_{m 0}$ increases accordingly. Concerning the leading edge flap, its contribution to $C_{m 0}$ depends on the sign of $\delta$, being positive when $\delta>0$ and negative when the flap is deflected downward $\delta<0$. Therefore, when $\delta>0$, both effects, camber and leading edge flap, contribute with the same sign to the pitching moment, whereas when $\delta<0$, they act in opposition, which explain the change in the $C_{m 0}$ versus $\delta$ curve slope.

The maximum efficiency, $\eta_{\max }$, data plotted in Figure 10 reveals that, depending on the Reynolds number, in the range $-0.0050 \leqslant \delta \leqslant 0.0025$, the maximum efficiency is almost constant or even decreases as $\delta$ grows, which seems to be the range of admissible leading edge deformations. Therefore, close enough to the nominal unperturbed geometry $(\delta=0)$, a cambered aerofoil like the NACA $63_{2}-215$ with these leading edge imperfections shows almost negligible aerodynamic performance degradation, although this behaviour is kept only in a very narrow interval 


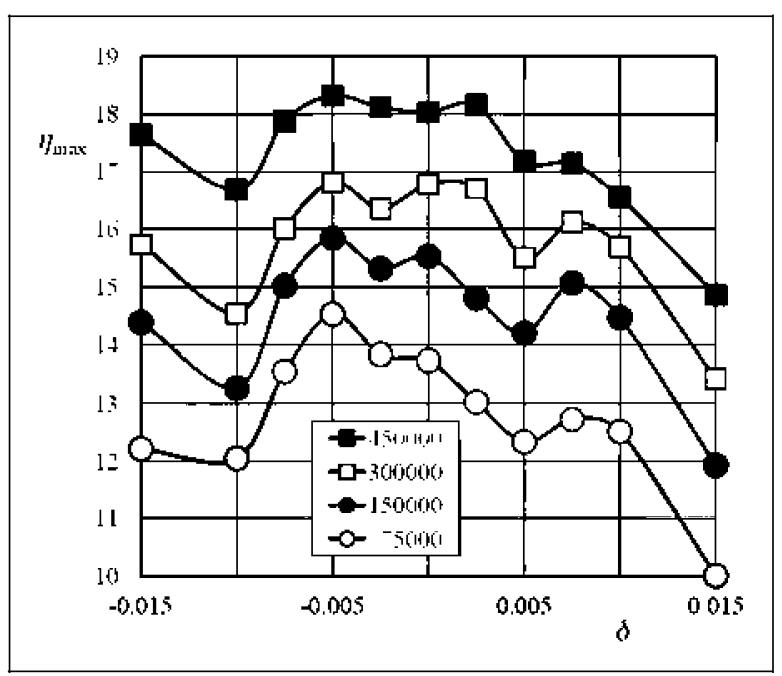

Figure 10. Variation with the leading edge displacement $\delta$ of the maximum efficiency, $\eta_{\max }=\left(C_{1} / C_{d}\right)_{\max }$, of modified NACA $63_{2}-215$ aerofoils. Symbols identify the value of the Reynolds number according to the following key: $\operatorname{Re}=0.75 \times 10^{5}$ (white circles); $1.5 \times 10^{5}$ (black circles); $3.0 \times 10^{5}$ (white squares) and $4.5 \times 10^{5}$ (black squares).

of $\delta$ values. Outside this interval, say $|\delta|>0.0050$, the drop of aerodynamic efficiency is larger than $5 \%$ of the nominal value and becomes unacceptable.

\section{Conclusions}

Leading edge imperfections can arise during the manufacturing process of aerofoils in some commercial applications. A typical example can be found in wind turbine blades, which are usually manufactured in two parts, or shells, and after assembled. Since wind turbines usually work in a rather turbulent environment (the atmospheric boundary layer), in our opinion, the test conditions used in aeronautical applications can be relaxed, which allows the use of wind tunnels with a flow with a turbulence intensity not so small than the one needed in aeronautical applications.

Experiments performed with NACA $63_{2}-215$ aerofoils whose leading edge is perturbed by a small displacement $\delta$ of the upper side half with respect to the lower one show that the maximum lift coefficient slightly increases when such a displacement between upper and lower aerofoil surfaces at the leading edge grows, provided $|\delta| \leqslant 0.0050$, this increment being noticeable at low Reynolds numbers when $\delta=0.0050$ (see the plots corresponding to $\mathrm{Re}=$ $0.75 \times 10^{5}$ and $\operatorname{Re}=1.5 \times 10^{5}$ in Figure 7).

Associated with the leading edge imperfection $\delta$, there is a remarkable increment of the aerodynamic drag and since the increase of aerodynamic drag is larger than the one of lift, the aerofoil efficiency decreases as the displacement grows.

The main conclusion that can be derived from the results presented above is that a small leading edge imperfection like the one here considered could be tolerated, provided the magnitude of such an imperfection, measured by the leading edge displacement parameter, $\delta$, be within small boundaries, $|\delta|>0.0050$.

\section{Funding}

This research received no specific grant from any funding agency in the public, commercial, or not-for-profit sectors.

\section{References}

1. Carmichael BH. Low Reynolds number airfoil survey. Report, NASA CR-165803, Vol. 1, 1981, USA.

2. Nagamatsu HT and Cuche DE. Low Reynolds number aerodynamics characteristics of low-drag NACA 63-208 airfoil. J Aircraft 1981; 18: 833-837.

3. Mueller TJ and Batill SM. Experimental studies of separation on a two-dimensional airfoil at low Reynolds number. AIAA $J$ 1982; 20: 457-463.

4. Lissaman PBS. Low-Reynolds-number airfoils. Ann Rev Fluids Mech 1983; 15: 223-239.

5. Cebeci $T$. Essential ingredients of a method for low Reynolds number airfoils. AIAA $J 1989 ; 27$ : 1680-1688.

6. Bloy AW and Roberts DG. Aerodynamic characteristics of the NACA 63(2)-215 aerofoil for use in wind turbines. Wind Eng 1993; 17: 67-75.

7. Mueller TJ and DeLaurier JD. Aerodynamics of small vehicles. Ann Rev Fluids Mech 2003; 35: 98-111.

8. Torres GE and Mueller TJ. Low-aspect-ratio wing aerodynamics at low Reynolds number. AIAA $J$ 2004; 42: 865-873.

9. Ayuso L, Sant R and Meseguer J. Aerodynamic study of airfoils with leading edge imperfections at low Reynolds number. In: 40th AIAA fluid dynamics conference, 2010, Chicago, IL, USA.

10. Sant R, Ayuso L and Meseguer J. Aerodynamic study of airfoil geometric imperfections at low Reynolds number. In: 13th international conference on wind engineering, ICWE13, Amsterdam, The Netherlands, 2011.

11. Jones AR, Bakhtian NM and Babinsky H. Low Reynolds number aerodynamics of leading-edge flaps. $J$ Aircraft 2008; 45: 342-345.

12. Abbott IH and Von Doenhoff AE. Theory of wing sections. NY, USA: Dover Publications Inc, 1959.

13. Allen HJ and Vincenti WG. Wall interference in a twodimensional-flow wind tunnel, with consideration of compressibility. NACA Report no. 782, 1944, USA.

14. Pope A and Harper JJ. Low speed wind tunnel testing. New York, USA: Wiley, 1966.

15. Marchman JF. Aerodynamics testing at low Reynolds numbers. $J$ Aircraft 1987; 24: 107-114.

16. McGullough GB and Gault DE. Examples of three representative types of airfoil-section stall at low speed, NACA Report no. TN 2502, 1951, USA.

17. Palme HO. Summary of stalling characteristics and maximum lift of wings at low speed, SAAB TN 15, Svenska Aeroplan Akbtiebolaget, Linköping, Sweden, 1953.

\section{Appendix}

\section{Notation}

C nominal aerofoil chord length 


$\begin{array}{llll}C_{d} & \text { drag coefficient } & U_{\min } & \text { test chamber minimum velocity } \\ C_{l} & \text { lift coefficient } & U_{\infty} & \text { test chamber air speed } \\ C_{m} & \text { pitch moment coefficient } & x_{d} & \text { leading edge displacement } \\ c \delta & \text { modified aerofoil chord length } & \alpha & \text { aerodynamic angle of attack } \\ d & \text { drag force } & \delta & \text { dimensionless leading edge } \\ l & \text { lift force } & & \text { displacement } \\ m & \text { pitch moment } & \eta & \text { aerodynamic efficiency } \\ q_{\infty} & \text { dynamic pressure } & \eta_{\max } & \text { maximum aerodynamic efficiency } \\ \operatorname{Re} & \text { Reynolds number } & \rho & \text { air density } \\ U_{\text {max }} & \text { test chamber maximum velocity } & & \\ U_{\text {mean }} & \text { test chamber mean velocity } & & \end{array}$

